\title{
Erratum: Numerical study on collective excitations in graphene [Phys. Rev. B 94, 155451 (2016)]
}

Seiji Uryu

(Received 4 November 2017; published 27 November 2017)

DOI: 10.1103/PhysRevB.96.199906

In our earlier work, there was an error in the numerical calculations. We correct the related numerical results. The correction leads to changes in the wave-vector dependences of the conductivity in Fig. 3(a), the transition matrix elements in Figs. 4(a), 4(b), and 6(b), and the plasmon peak intensity in Figs. 5(a), 5(b), 6(c), and 10(b), where the figure numbers refer to those of the earlier work. For small wave vectors, the transition matrix elements are enhanced as compared to the earlier results and the wave-vector dependences of the plasmon peak intensity change from increasing functions to decreasing functions. However, these changes do not affect any of the conclusions of the earlier work.

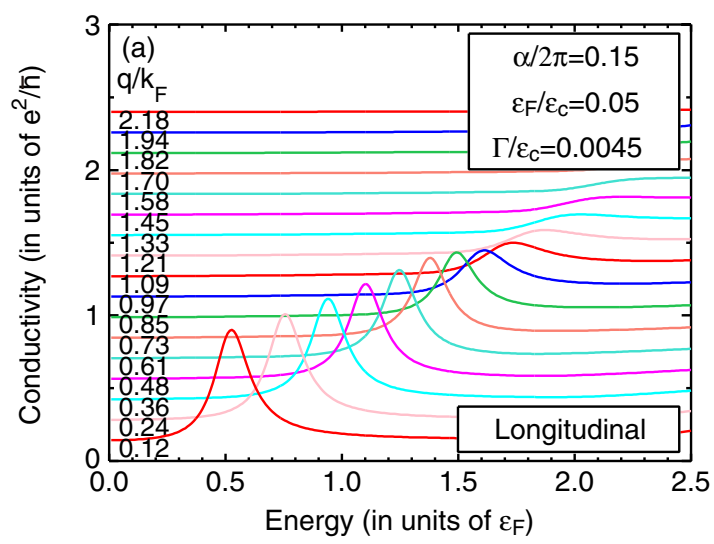

FIG. 3. (a) Real parts of the longitudinal component of the dynamical conductivity for various wave vectors q. Phenomenological energy broadening is $\Gamma / \varepsilon_{c}=0.0045$.
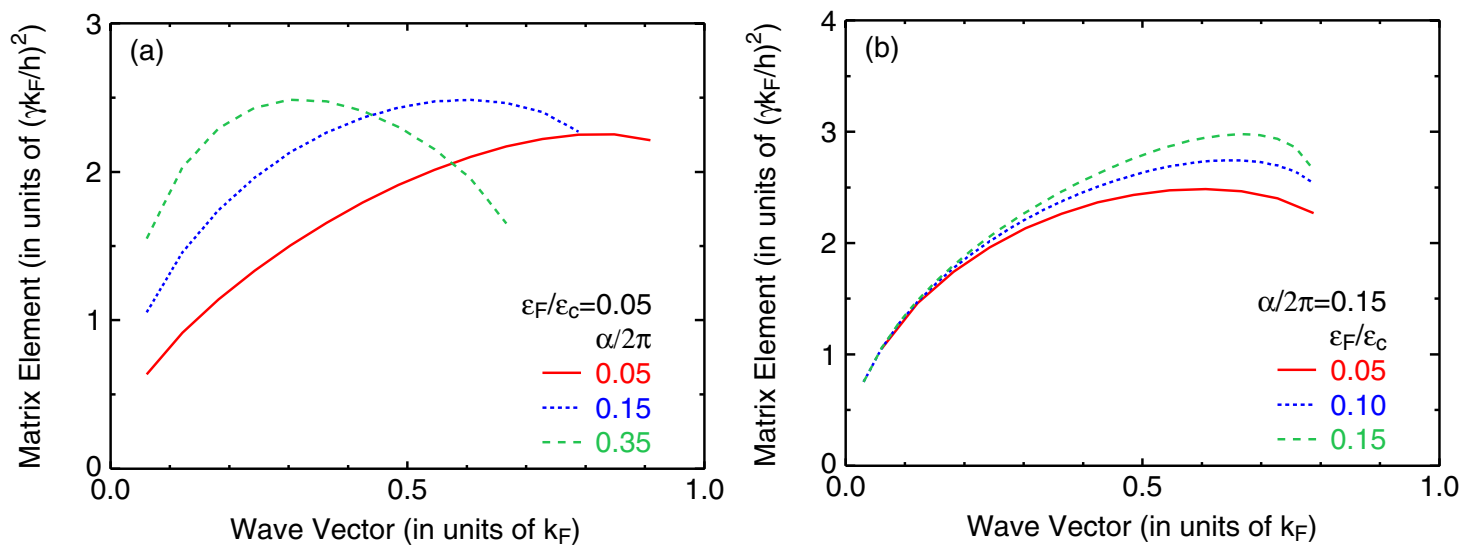

FIG. 4. Squared absolute values of the velocity matrix elements $\left|\left\langle v \mathbf{q}\left|v_{\beta}\right| G\right\rangle\right|^{2} / L^{2}$ for plasmons for various values of (a) the Coulomb interaction parameter and (b) the Fermi energy. In panel (a), $\alpha / 2 \pi=0.05$ (solid line), 0.15 (dotted line), and 0.35 (dashed line) for $\varepsilon_{F} / \varepsilon_{c}=0.05$. In panel (b), $\varepsilon_{F} / \varepsilon_{c}=0.05$ (solid line), 0.1 (dotted line), and 0.15 (dashed line) for $\alpha / 2 \pi=0.15$. 

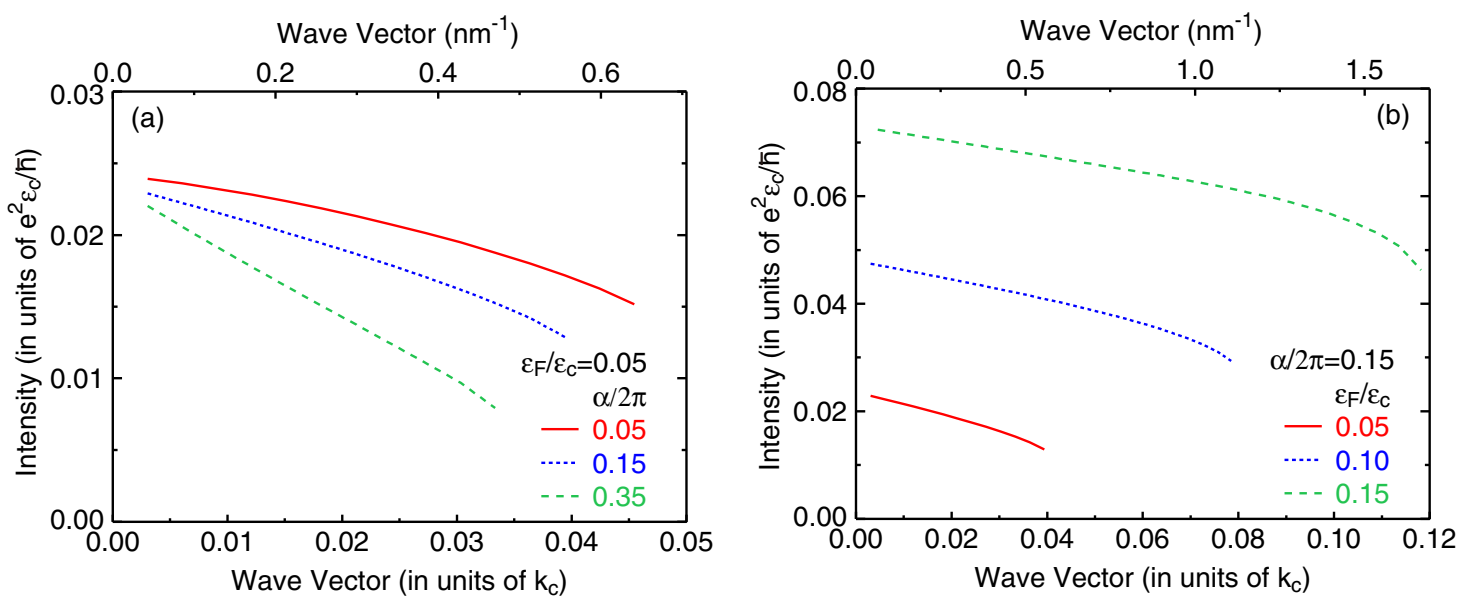

FIG. 5. Intensity of plasmon peaks in absorption spectra for various values of (a) the Coulomb interaction parameter and (b) the Fermi energy.
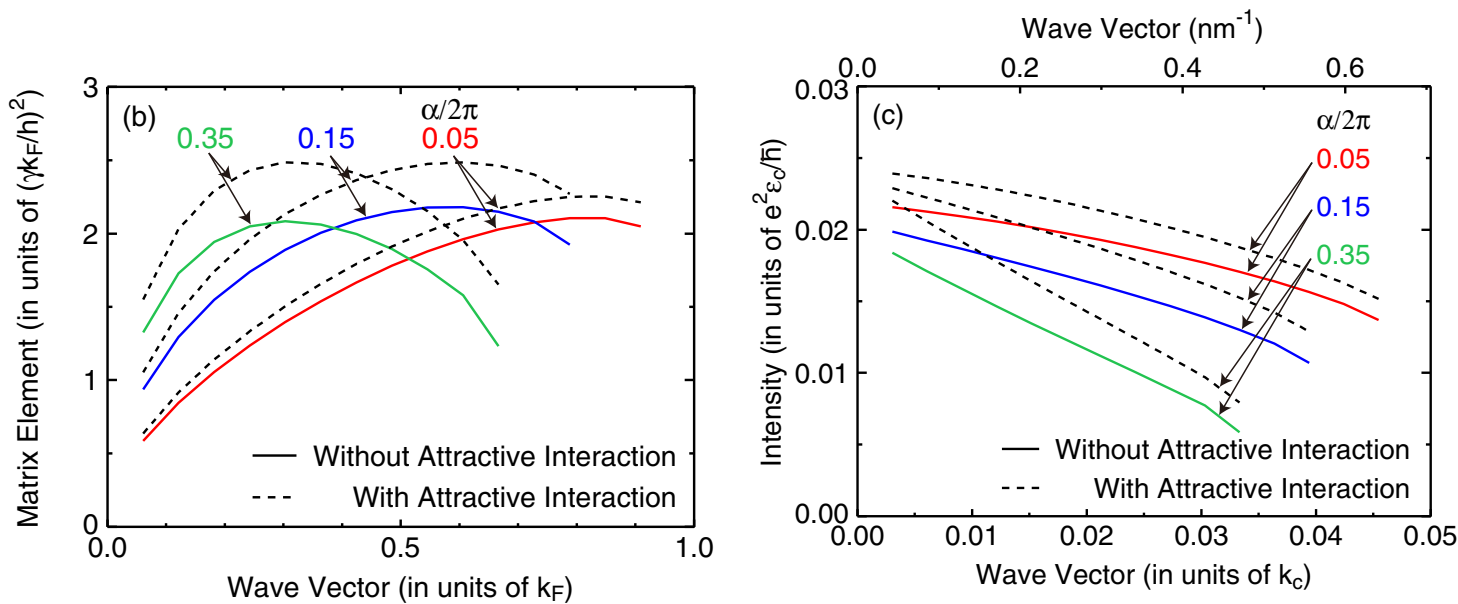

FIG. 6. (b) Squared absolute values of the velocity matrix elements and (c) intensity of peaks in absorption spectra for plasmons. Solid and dashed lines are results calculated without and with the e-h attractive interactions, respectively.

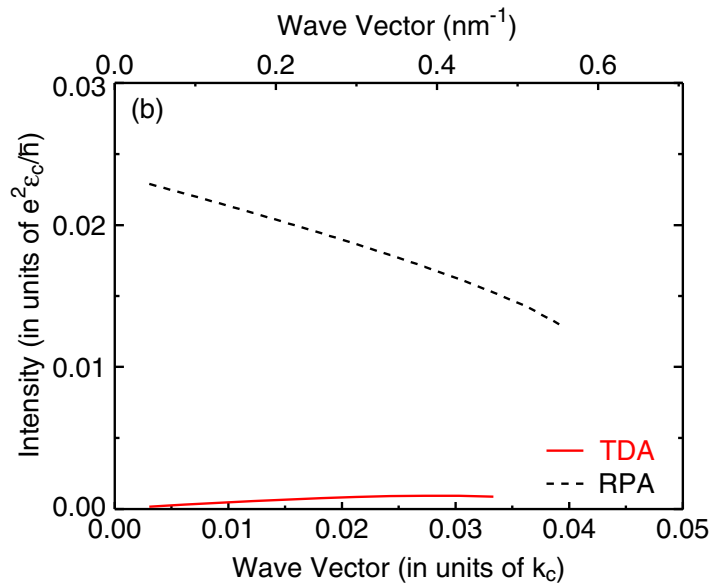

FIG. 10. (b) Absorption peak intensity for plasmons in doped graphene with $\alpha / 2 \pi=0.15$ and $\varepsilon_{F} / \varepsilon_{c}=0.05$. Solid and dashed lines indicate results calculated in TDA and RPA, respectively. 